\title{
TRADUCTOR MÓVIL PARA ASISTENCIA MÉDICA A PACIENTES QUECHUA
}

MOBILE TRANSLATOR FOR MEDICAL ASSISTANCE FOR QUECHUA SPEAKING PATIENTS

Manuel Avelino Lagos Barzola ${ }^{1}$

Zuliana Villar Vila

1. Profesor del Dpto. de Matemática y Física, Área de Informática. Universidad Nacional de San Cristóbal de Huamanga, Ayacucho (Perú).E-mail: manuel.lagos@unsch.edu.pe

2. Bióloga por la Universidad Nacional de San Cristóbal de Huamanga, Ayacucho. Médico por la Universidad Científica del Sur, Lima (Perú). E-mail: zvillarv@hotmail.com

\section{Citación sugerida:}

Lagos Barzola, M.A. y Villar Vila, Z. (2017). Traductor móvil para asistencia médica a pacientes quechua hablantes. 3C Tecnología: glosas de innovación aplicadas a la pyme, 6(1), 15-34. DOI: <http://dx.doi.org/10.17993/3ctecno.2017.v6n1e21.15-34/>. 


\section{RESUMEN}

El propósito del trabajo es desarrollar un software traductor móvil que permita realizar un diagnóstico adecuado a pacientes quechua hablantes a través de una comunicación fluida, en las instituciones de salud de la región Ayacucho. El caso de estudio se desarrolló en el Centro de Salud de Carmen Alto de Ayacucho, y el software fue diseñado para ser utilizado en atención de consultorio de medicina general.

Para el desarrollo del traductor móvil se utilizó el proceso ágil de desarrollo de Software de Programación Extrema. Para determinar la validez del instrumento desarrollado, se utilizó el diseño de validación y confiabilidad del instrumento. La validez del software se sustenta en que en su desarrollo se tuvo la participación de expertos en salud.

El trabajo de investigación se realizó en dos etapas tras la construcción del software. En la primera se realizó un análisis de fiabilidad del software y se determinó si el software permitía al médico realizar preguntas y registrar los síntomas de los pacientes quechua hablantes. En la segunda etapa se determinó el resultado del nivel de concordancia entre el diagnóstico de dichos pacientes con la colaboración de dos médicos: uno con dominio y fluidez del quechua, mientras que el segundo no. Se debe toner en cuenta que para este último se usó la herramienta desarrollada.

Finalmente, con un alto nivel de confianza, se colige que el traductor móvil para asistencia médica a pacientes quechua hablantes permite al médico general realizar preguntas satisfactoriamente y registrar los síntomas de sus pacientes. Además, con un índice de Kappa de Cohen de 0,804, se precisa que la herramienta es altamente confiable para el diagnóstico de los pacientes con la característica idiomática requerida.

\section{ABSTRACT}

The purpose of this research is the development of a mobile translator software that allows a medical diagnosis to Quechua speaking patients, through a fluently communication in the health institutions of the region of Ayacucho. The case of study was developed at Carmen Alto Health Center in Ayacucho, and the software was designed to be used just in general medicine.

To create the mobile translator, it was used the agile process of extreme programming software development. On the other hand, in order to determine the validity of the developed instrument, it was used the validation and reliability design of the instrument. The validity of the software is based on the fact that its development has involved the participation of health experts.

The application work was taken place in two stages. In the first staage, a reliability analysis of the software was performed and it was determined if the software allowed the doctor to ask the questions and record the symptoms of the Quechua speaking patients. In the second stage, the result of the level of concordance between the diagnosis of the patients was held with the help of two doctors: one who mastered Quechua, while the second one did not. It should be taken into account that the second doctor used the developed tool.Finally, through a high level of trust, it is concluded that the mobile translator for medical care to Quechua speaking patients allows the general practitioner to ask questions satisfactorily and record the symptoms of their patients. In addition, with a Cohen Kappa index of 0.804 , it is specified that the tool is highly reliable for the diagnosis of patients with the required idiomatic feature. 


\section{PALABRAS CLAVE}

Traductor móvil, asistencia médica, paciente quechua hablante, medicina general.

\section{KEY WORDS}

Mobile translator, medical assistance, quechua speaking patient, general medicine. 


\section{INTRODUCCIÓN}

Con respecto a la calidad de la atención médica, Donabedian, (1984) refiere que es "el grado en que los medios más deseables se utilizan para alcanzar las mayores mejoras posibles en la salud". Propone tres dimensiones involucradas para lograr la calidad de la atención médica: la dimensión estructura, la dimensión proceso y la dimensión resultados. En la segunda dimensión, como componente importante, menciona el proceso de comunicación.

El tema de investigación es el desarrollo de un traductor móvil para asistencia médica a pacientes quechua hablantes. El fundamento para realizar esta investigación fue la problemática que sufre la comunidad de quechua hablantes de la región de Ayacucho, ya que la asistencia médica es deficiente en zonas rurales o de bajos recursos, la cual se plasma en inadecuados diagnósticos y comunicación entre el médico hispanohablante y el paciente quechua hablante. Cabe resaltar que esta problemática es poco conocida por la falta de denuncias de estos pacientes.

Se desarrolla el traductor móvil de asistencia médica con el objetivo principal de brindar una herramienta al sector salud, en especial los médicos para que puedan realizar un diagnóstico adecuado a pacientes quechua hablantes, a través de una comunicación fluida.

Se desarrolló el traductor móvil bajo el proceso ágil de Programación Extrema y, finalmente, se validó la confiabilidad del software para el diagnóstico de los pacientes por parte de los médicos no quechua hablantes.

\section{METODOLOGÍA}

\subsection{DISEÑO DE LA INVESTIGACIÓN}

Según Carrasco, (2005) el diseño de investigación no experimental de tipo transversal se emplea para analizar y conocer las características, rasgos, propiedades y cualidades de un hecho realizado en un momento determinado de tiempo.

Según el punto anterior, el diseño de investigación para el trabajo de tesis es no experimental de tipo transversal, debido a que se busca recoger la información de forma directa en un momento determinado. Se tiene como objetivo básicamente el de precisar y describir de manera pertinente el software traductor de asistencia médica para pacientes quechua hablantes, así como verificar su confiabilidad en el diagnóstico médico. Por otro lado, para determinar la validez del instrumento tipo software desarrollado se utilizó el diseño de validación y confiabilidad del instrumento.

\subsection{POBLACIÓN}

Pacientes que acuden al Centro de Salud de Carmen Alto, Ayacucho y quienes padecen de enfermedades comunes como faringitis aguda, amigdalitis aguda, bronquiolitis y neumonía. 


\subsection{POBLACIÓN MUESTRAL}

Son los resultados de los diagnósticos obtenidos a partir de la evaluación con el software usado por el médico no quechua hablante y sin el software, pero con la presencia de un médico quechua hablante a los pacientes mencionados con antelación.

\subsection{MUESTRA}

Primero, para saber si el software permitía realizar preguntas satisfactoriamente a pacientes quechua hablantes para así registrar sus síntomas, se hizo la prueba en una muestra intencionada de setenta y cinco pacientes que asistieron al Centro de Salud de Carmen Alto, Ayacucho.

Segundo, para ver si el software demostraba efectividad en el diagnóstico de los pacientes, se hizo la prueba en una muestra intencionada de noventa y seis pacientes en el Centro de Salud ya mencionado.

\subsection{TÉCNICAS E INSTRUMENTOS}

Las técnicas utilizadas para el estudio fueron:

a) Lista de cotejo

b) Análisis documental

Los instrumentos utilizados fueron:

a) Lista de cotejo

b) Ficha de historia clínica

\section{RESULTADOS Y ANÁLISIS DE RESULTADOS}

\subsection{DESARROLLO DEL SOFTWARE}

\section{Proceso de atención de pacientes en consultorio de medicina general}

Regularmente en un consultorio de medicina general el proceso de atención a pacientes por parte del médico sigue el siguiente flujograma: 


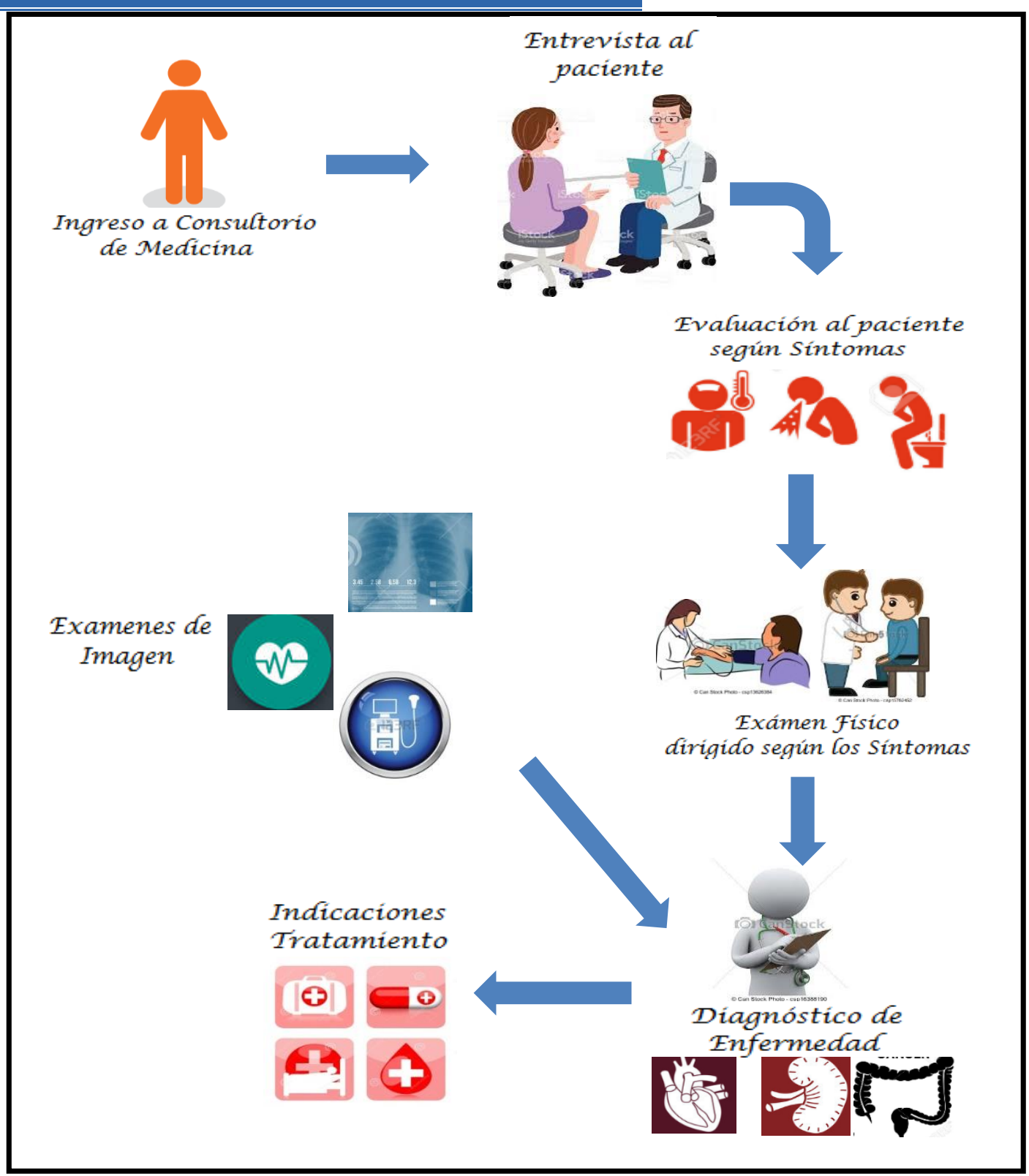

Gráfico 1. Flujograma del proceso de atención en consultorio de medicina general.

Fuente: Elaboración propia.

La aplicación móvil de asistencia médica para pacientes quechua hablantes se construyó como herramienta de apoyo para el proceso de comunicación en estas actividades.

Sobre la elección del proceso de desarrollo de Software para el traductor móvil

Si tuviésemos que responder a la pregunta: ¿por qué elegir un proceso de desarrollo de software ágil?

Nuestra respuesta está muy de acuerdo con lo que manifiestó Jacobson (2002) y que Pressman (2010) hace referencia, respecto a la agilidad: 
La agilidad se ha convertido en la palabra mágica de hoy día para describir un proceso del software moderno. Todos son ágiles. Un equipo ágil es diestro y capaz de responder de manera apropiada a los cambios. El cambio es de lo que trata el software en gran medida. Hay cambios en el Software que se construyen en los miembros del equipo debidos a las nuevas tecnologías, de todas clases y que tienen un efecto en el producto que se elabora o en el proyecto que lo crea. Deben introducirse apoyos para el cambio en todo lo que se haga en el software y en ocasiones se hace porque es alma y corazón de éste. Un equipo ágil reconoce que el software es desarrollado por individuos que trabajan en equipo, y que su capacidad, su habilidad para colaborar, es el fundamento para el éxito del proyecto (p. 88).Y por otro lado, es imperiosa la necesidad de abordar temas como el que precisó Fowler, que recoge también Pressman (2010):

a) Es difícil predecir qué requerimientos de software persistirán y cuáles cambiarán. También es difícil pronosticar cómo cambiarán las prioridades del cliente a medida que avanza el proyecto.

b) Para muchos tipos de software, el diseño y la construcción están imbricados. Es decir, ambas actividades deben ejecutarse en forma simultánea, de modo que los modelos de diseño se prueban a medida que se crean. Es difícil predecir cuánto diseño se necesita antes de que se use la construcción para probar el diseño.

c) En análisis, el diseño, la construcción y las pruebas no son tan predecibles como nos gustaría (desde el punto de vista de planeación).

Así mismo, se buscó alcanzar principios de agilidad como: que nuestra prioridad fue la de satisfacer al cliente a través de entregas prontas del software. Fueron bienvenidos los requerimientos cambiantes, se entregó con frecuencia software funcional, se trabajó junto con los clientes (médicos) y la conversación fue cara a cara.

Finalmente, y con respecto a la elección del proceso de desarrollo de software de Programación Extrema se dio gracias a las siguientes razones:

a) En primer lugar, cumple con las expectativas de agilidad de un proceso ágil de desarrollo de Software requeridos en este proyecto.

b) Pressman, (2010) afirma que es el enfoque más utilizado del desarrollo de software ágil. Consultada otras fuentes Scrum/XP Hybrid es la segunda más utilizada, frente a Scrum que es la más utilizada (http://bspin.org/, 2012).

c) Y el volumen promedio de bibliografía del uso de la Programación Extrema, frente a SCRUM y otros procesos ágiles.

\section{Desarrollo de la implementación del Software}

La implementación del software siguió las etapas del proceso de desarrollo de software de Programación Extrema: exploración, planeación, iteraciones a la primera versión y puesta en producción. 
Las historias de usuario obtenidas por parte del personal médico fueron:

Tabla 1. Historia de usuario realizar preguntas al paciente en quechua.

\begin{tabular}{|c|c|}
\hline \multicolumn{2}{|c|}{ Historia de usuario } \\
\hline Nombre: Rea & al paciente en quechua. \\
\hline \multicolumn{2}{|l|}{ Usuario: Médico } \\
\hline Modificación de Historia $\mathrm{N}^{\circ}$ : NA & Iteración asignada: 1 \\
\hline Prioridad de negocio: Alta & Riesgo en desarrollo: Media \\
\hline $\begin{array}{l}\text { Descripción: } \\
\text { Como médico quiero realizar } \\
\text { hablante acerca de sus síntoma } \\
\text { para realizar su diagnóstico. }\end{array}$ & $\begin{array}{l}\text { cir órdenes en quechua al paciente quechua } \\
\text { de obtener información crítica a ser analizada }\end{array}$ \\
\hline
\end{tabular}

Fuente: elaboración propia.

Tabla 2. Dar alternativas de respuesta en quechua al paciente.

\begin{tabular}{|c|c|}
\hline \multicolumn{2}{|c|}{ Historia de usuario } \\
\hline Número: 2 & de respuesta en quechua al paciente. \\
\hline \multicolumn{2}{|l|}{ Usuario: Médico } \\
\hline Modificación de Historia $\mathrm{N}^{\circ}$ : NA & Iteración asignada: 1 \\
\hline Prioridad de negocio: Alta & Riesgo en desarrollo: Media \\
\hline $\begin{array}{l}\text { Descripción: } \\
\text { Como médico quiero brindar in } \\
\text { realizada al paciente quechua } \mathrm{h} \\
\text { de respuesta. }\end{array}$ & $\begin{array}{l}\text { las alternativas de respuesta de cada pregunta } \\
\text { bjetivo de dar al paciente sólo alternativas fijas }\end{array}$ \\
\hline
\end{tabular}

Fuente: elaboración propia.

Tabla 3. Resumen de respuestas del paciente

\begin{tabular}{|l|l|}
\hline Número: 3 & Historia de usuario \\
\hline Usuario: Médico & Iteración asignada: 1 \\
\hline Modificación de Historia $N^{\circ}:$ NA & Riesgo en desarrollo: Media \\
\hline Prioridad de negocio: Alta & $\begin{array}{l}\text { Descripción: } \\
\text { Como médico quiero un resumen de todas las respuestas dadas por el paciente quechua } \\
\text { hablante, con el objetivo de tener un reporte resumido de toda la información brindada por el } \\
\text { paciente acerca de sus síntomas. }\end{array}$ \\
\hline
\end{tabular}

Fuente: elaboración propia. 
Tabla 4. Diagnosticar al paciente en quechua.

\begin{tabular}{|l|l|}
\hline Número:4 Nombre: Diagnosticar al paciente en quechua. \\
\hline Usuario: Médico \\
\hline Modificación de Historia $N^{\circ}:$ NA & Iteración asignada: 1 \\
\hline Prioridad de negocio: Alta & Riesgo en desarrollo: Media \\
\hline $\begin{array}{l}\text { Descripción: } \\
\text { Como médico quiero comunicar el diagnóstico de la enfermedad al paciente en quechua, con el } \\
\text { objetivo de que el paciente quechua hablante entienda qué enfermedad padece. }\end{array}$ \\
\hline
\end{tabular}

Fuente: elaboración propia.

Tabla 5. Dar tratamiento farmacológico en quechua al paciente.

\begin{tabular}{|c|c|}
\hline \multicolumn{2}{|c|}{ Historia de usuario } \\
\hline Nombre: Dar & macológico en quechua al paciente. \\
\hline \multicolumn{2}{|l|}{ Usuario: Médico } \\
\hline Modificación de Historia $\mathrm{N}^{\circ}$ : NA & Iteración asignada: 1 \\
\hline Prioridad de negocio: Alta & Riesgo en desarrollo: Media \\
\hline \multicolumn{2}{|c|}{$\begin{array}{l}\text { Descripción: } \\
\text { Como médico quiero comunicar el tratamiento farmacológico al paciente en quechua, con el } \\
\text { objetivo de que el paciente quechua hablante entienda qué medicamentos tomar, así como la } \\
\text { duración y frecuencia del mismo. }\end{array}$} \\
\hline
\end{tabular}

Fuente: elaboración propia.

Tabla 6. Dar tratamiento preventivo en quechua al paciente.

\begin{tabular}{|c|c|}
\hline \multicolumn{2}{|c|}{ Historia de usuario } \\
\hline Nombre: Dar & ventivo en quechua al paciente. \\
\hline \multicolumn{2}{|l|}{ Usuario: Médico } \\
\hline Modificación de Historia $\mathrm{N}^{\circ}$ : NA & Iteración asignada: 1 \\
\hline Prioridad de negocio: Alta & Riesgo en desarrollo: Media \\
\hline $\begin{array}{l}\text { Descripción: } \\
\text { Como médico quiero comunic } \\
\text { objetivo de que el paciente quec } \\
\text { diagnóstico. }\end{array}$ & $\begin{array}{l}\text { preventivo al paciente en quechua, con el } \\
\text { ienda qué acciones preventivas realizar para su }\end{array}$ \\
\hline
\end{tabular}

Fuente: elaboración propia. 


\section{Tecnologías utilizadas para el desarrollo del software traductor móvil}

Las herramientas tecnológicas que se utilizaron, son en gran parte de software libre, fueron seleccionadas en función a las necesidades y limitaciones como recursos económicos, humanos y el tipo de aplicación que es el software traductor móvil de asistencia médica.

Tabla 7. Herramientas de desarrollo del traductor móvil de asistencia médica.

\begin{tabular}{|c|c|c|}
\hline \multicolumn{3}{|c|}{ Herramientas de desarrollo } \\
\hline SOFTWARE & FABRICANTE & DESCRIPCIÓN \\
\hline $\begin{array}{l}\text { MICROSOFT } \\
\text { WINDOWS } 8.1\end{array}$ & Microsoft & Sistema Operativo \\
\hline JAVA JDK & $\begin{array}{l}\text { Desarrollado } \\
\text { por Sun } \\
\text { Microsystems } \\
\text { originalmente }\end{array}$ & $\begin{array}{l}\text { Java es un lenguaje de programación orientado a } \\
\text { objetos, desarrollado por Sun Microsystems } \\
\text { originalmente, que posee un sistema que } \\
\text { interpreta y ejecuta los archivos para ser } \\
\text { compilados en tiempo real. }\end{array}$ \\
\hline ANDROID SDK & Google. & $\begin{array}{l}\text { El SDK (Software Development Kit) de Android, } \\
\text { incluye un conjunto de herramientas de desarrollo. } \\
\text { Comprende un depurador de código, biblioteca, un } \\
\text { simulador de teléfono basado en QEMU, } \\
\text { documentación, ejemplos de código y tutoriales. } \\
\text { Las plataformas de desarrollo soportadas incluyen } \\
\text { GNU/Linux, Mac OS X } 10.5 .8 \text { o posterior, y Windows } \\
\text { XP o posterior. }\end{array}$ \\
\hline ANDROID STUDIO & Google. & $\begin{array}{l}\text { Entorno de desarrollo integrado para la plataforma } \\
\text { Android. Está basado en el software IntelliJ IDEA de } \\
\text { JetBrains, y es publicado de forma gratuita a través } \\
\text { de la Licencia Apache 2.0. Está disponible para las } \\
\text { plataformas Microsoft Windows, Mac OS X y } \\
\text { GNU/Linux. }\end{array}$ \\
\hline SQLite & Richard & $\begin{array}{l}\text { Sistema de gestión de bases de datos relacional } \\
\text { compatible con } A C I D \text {, contenida en una } \\
\text { relativamente pequeña ( } \sim 275 \mathrm{kB} \text { ) biblioteca escrita } \\
\text { en C. SQLite es un proyecto de dominio público. }\end{array}$ \\
\hline $\begin{array}{l}\text { https://www.mat } \\
\text { erialpalette.com/ }\end{array}$ & - & $\begin{array}{l}\text { Herramienta en línea para trabajar combinaciones } \\
\text { de colores basados Google Material Design. }\end{array}$ \\
\hline $\begin{array}{l}\text { https://www.flui } \\
\text { dui.com }\end{array}$ & - & $\begin{array}{l}\text { Herramienta en línea para elaboración de } \\
\text { prototipos de aplicaciones móviles así como el } \\
\text { flujograma de la aplicación. }\end{array}$ \\
\hline$\frac{\mathrm{https}: / / \text { icons8.co }}{\mathrm{m} / \mathrm{L}}$ & - & $\begin{array}{l}\text { Herramienta en línea que brinda un conjunto rico } \\
\text { de íconos. }\end{array}$ \\
\hline
\end{tabular}

Fuente: elaboración propia. 


\section{Herramientas para la ejecución del software traductor móvil de asistencia médica}

Son necesarias las siguientes herramientas para que el usuario pueda ejecutar la aplicación móvil.

Tabla 8. Herramientas para la ejecución del traductor móvil de asistencia médica.

\begin{tabular}{|lll|}
\hline SOFTWARE & \multicolumn{2}{c|}{ Herramientas para ejecución } \\
\hline $\begin{array}{l}\text { SISTEMA } \\
\text { OPERATIVO }\end{array}$ Google. & \multicolumn{1}{c|}{ DESCRIPCIÓN } \\
ANDROID & & $\begin{array}{l}\text { Sistema operativo basado en el núcleo Linux. } \\
\text { Fue diseñado principalmente para dispositivos } \\
\text { móviles con pantalla táctil, como teléfonos } \\
\text { inteligentes, tablets o tabléfonos; y también } \\
\text { para relojes inteligentes, televisores y } \\
\text { automóviles }\end{array}$ \\
\hline SQLITE & D. Richard Hipp. & $\begin{array}{l}\text { Sistema de gestión de bases de datos } \\
\text { relacional compatible con ACID, contenida en } \\
\end{array}$ \\
& una relativamente pequeña ( 275 kB) \\
& biblioteca escrita en C. SQLite es un proyecto \\
& de dominio público. \\
\hline
\end{tabular}

Fuente: elaboración propia.

La interfaz gráfica del usuario está distribuida bajo la siguiente estructura:

1. Interfaz principal. A partir de esta interfaz se pueden acceder a las diferentes categorías de la aplicación web, como son:

a. Antecedentes. Categoría que permitirá al médico obtener información acerca de los antecedentes del paciente.

b. Síntomas. Categoría que permitirá al médico obtener información de los síntomas del paciente.

c. Examen físico. Categoría que permitirá al médico dar indicaciones para realizar el examen físico correspondiente al paciente.

d. Examen laboratorio. Categoría que permitirá al médico solicitar exámenes de laboratorio al paciente.

e. Examen de imagen. Categoría que permitirá al médico solicitar exámenes de tipo imagen al paciente.

f. Diagnóstico. Categoría que permitirá al médico precisar el diagnóstico del paciente.

g. Tratamiento. Categoría que permitirá al médico precisar el tratamiento farmacológico y preventivo al paciente. 
h. Preguntas comprobación. Categoría que permitirá al médico realizar algunas preguntas que le permita saber si el paciente entendió su diagnóstico y/o tratamiento.

i. Personal. Categoría orientada a que el usuario pueda crear sus propias preguntas, así como grabar sus propios audios.

j. Reporte. Categoría en la que el usuario podrá generar un reporte de las preguntas realizadas con las respectivas respuestas del paciente, registradas por el médico.

2. Interfaz categoría. Es la interfaz que nos muestra las subcategorías de la categoría seleccionada previamente, así, se muestran a continuación las subcategorías por cada categoría respectivamente:

a. Antecedentes
i. Antecedentes generales
ii. Antecedentes gineco - obstétrico
iii. Aparato cardiovascular
iv. Aparato respiratorio
v. Aparato urogenital
vi. Aparato digestivo
vii. Hematología y oncología
viii. Endocrinología
ix. Aparato locomotor
x. Neurológicas
xi. Infecciones
xii. Salud mental
xiii. Cabeza y cuello
xiv. Antecedentes quirurgicos
$x v$. Antecedente de vacunación

b. Sintomas
i. Sintomas generales
ii. Oido
iii. Buco-dental
iv. Ojo
v. Nariz
vi. Aparato respiratorio
vii. Aparato cardiovascular
viii. Aparato digestivo
ix. Aparato urinario
x. Aparato genital
xi. Mama
xii. Sistema nervioso
xiii. Piel
xiv. Salud mental

c. Examen físico

i. No presenta sub categorías 
d. Examen laboratorio

i. No presenta sub categorías

e. Examen de imagen

i. No presenta sub categorías

f. Diagnóstico
i. Oido
ii. Vista
iii. Piel
iv. Aparato cardiovascular
v. Aparato respiratorio
vi. Aparato digestivo
vii. Aparato locomotor
viii. Sistema nervioso
ix. Endocrinológicos
$x$. Salud mental
xi. Sangre
xii. Infecciones

g. Tratamiento
i. Tratamiento farmacológico
ii. Tratamiento preventivo
iii. Curaciones
iv. Duracion
v. Frecuencia

h. Preguntas comprobación

i. No presenta sub categorías

i. Personal

j. Reporte

i. No presenta sub categorías

\subsection{VALIDEZ DE CONTENIDO DEL SOFTWARE}

Se garantiza la validez de contenido del software para el diagnóstico médico a pacientes quechua hablantes, ya que sus requisitos funcionales fueron trabajados con expertos en el sector salud. Cabe precisar que el software está orientado a las consultas de medicina general.

\subsection{ANÁLISIS DE FIABILIDAD DEL SOFTWARE}

Los resultados del análisis de fiabilidad del software se basan en dos etapas y dos muestras intencionadas.

Primero: resultados de pruebas realizadas en pacientes para determinar si el software permitió realizar preguntas y registrar los síntomas de los pacientes 
Para saber si el software permitió realizar preguntas a pacientes, se hizo la prueba en una muestra intencionada de 75 pacientes y se obtuvo los resultados siguientes.

Tabla 9. Resultados de pruebas realizadas en pacientes para determinar si el software permitió realizar preguntas en el Centro de Salud de Carmen Alto.

\begin{tabular}{|c|c|c|c|}
\hline \multicolumn{2}{|c|}{} & Frecuencia & Porcentaje \\
\hline \multirow{2}{*}{ Válido } & No & 2 & 2,7 \\
\cline { 2 - 4 } & Sí & 73 & 97,3 \\
\cline { 2 - 4 } & Total & 75 & 100,0 \\
\hline
\end{tabular}

Fuente: elaboración propia.

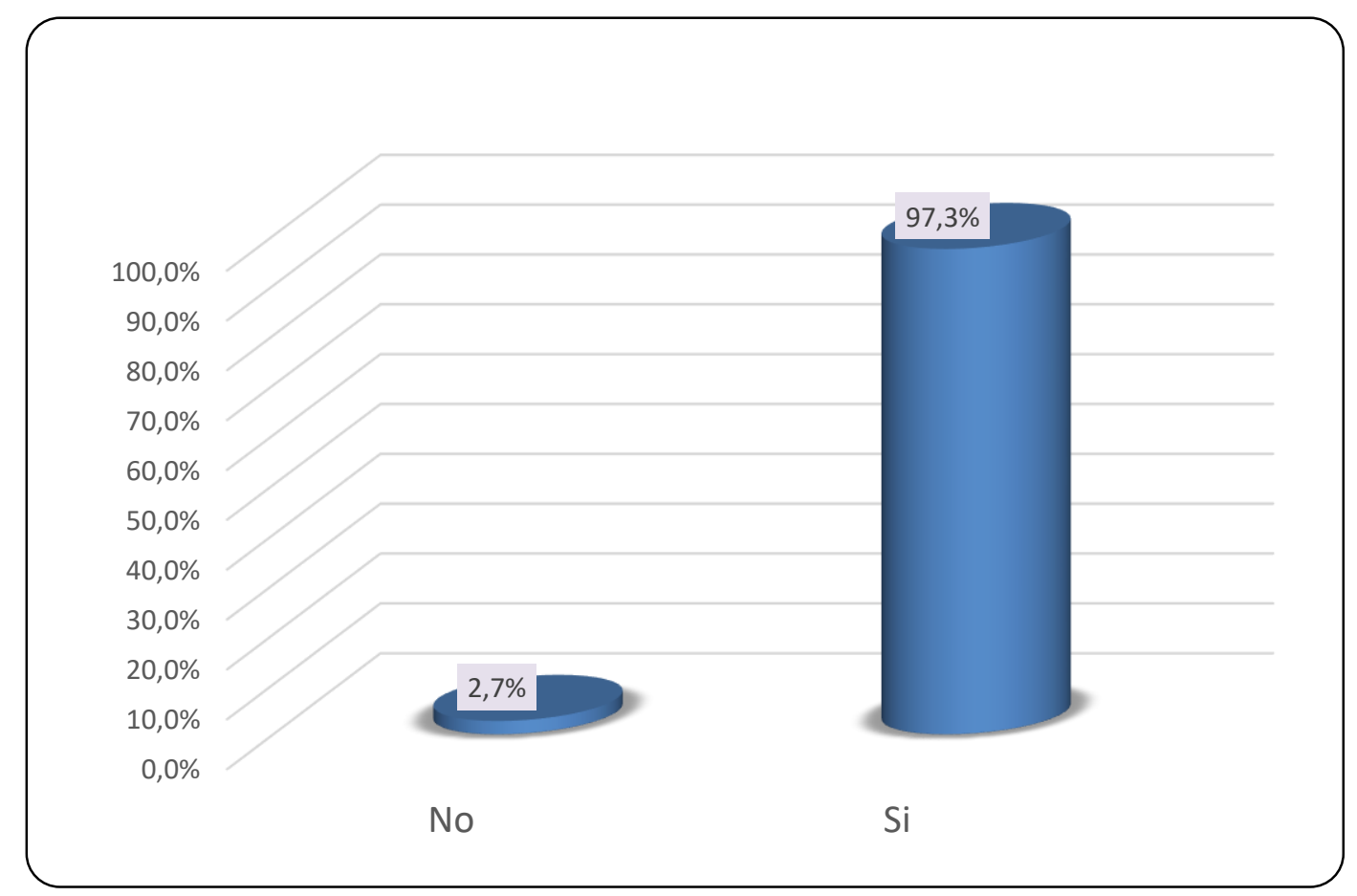

Gráfico 2. Resultados de pruebas realizadas en pacientes para determinar si el software permitió realizar preguntas en el Centro de Salud de Carmen Alto.

Fuente: elaboración propia.

Se observa que en el $97.3 \%$ de los pacientes, el software permitió realizar preguntas satisfactoriamente, hecho que indica que existe un alto nivel de confianza para poder efectuar preguntas a los pacientes.

Asimismo, para determinar si el software permite registrar los síntomas de los pacientes, se utilizó la misma muestra de la prueba anterior. 
Tabla 10. Resultados de pruebas realizadas en pacientes para determinar si el software permitió realizar preguntas en el Centro de Salud de Carmen Alto.

\begin{tabular}{|c|c|c|c|}
\hline \multicolumn{2}{|c|}{} & Frecuencia & Porcentaje \\
\hline \multirow{2}{*}{ Válido } & No & 2 & 2,7 \\
\cline { 2 - 4 } & Sí & 73 & 97,3 \\
\cline { 2 - 4 } & Total & 75 & 100,0 \\
\hline
\end{tabular}

Fuente: elaboración propia.

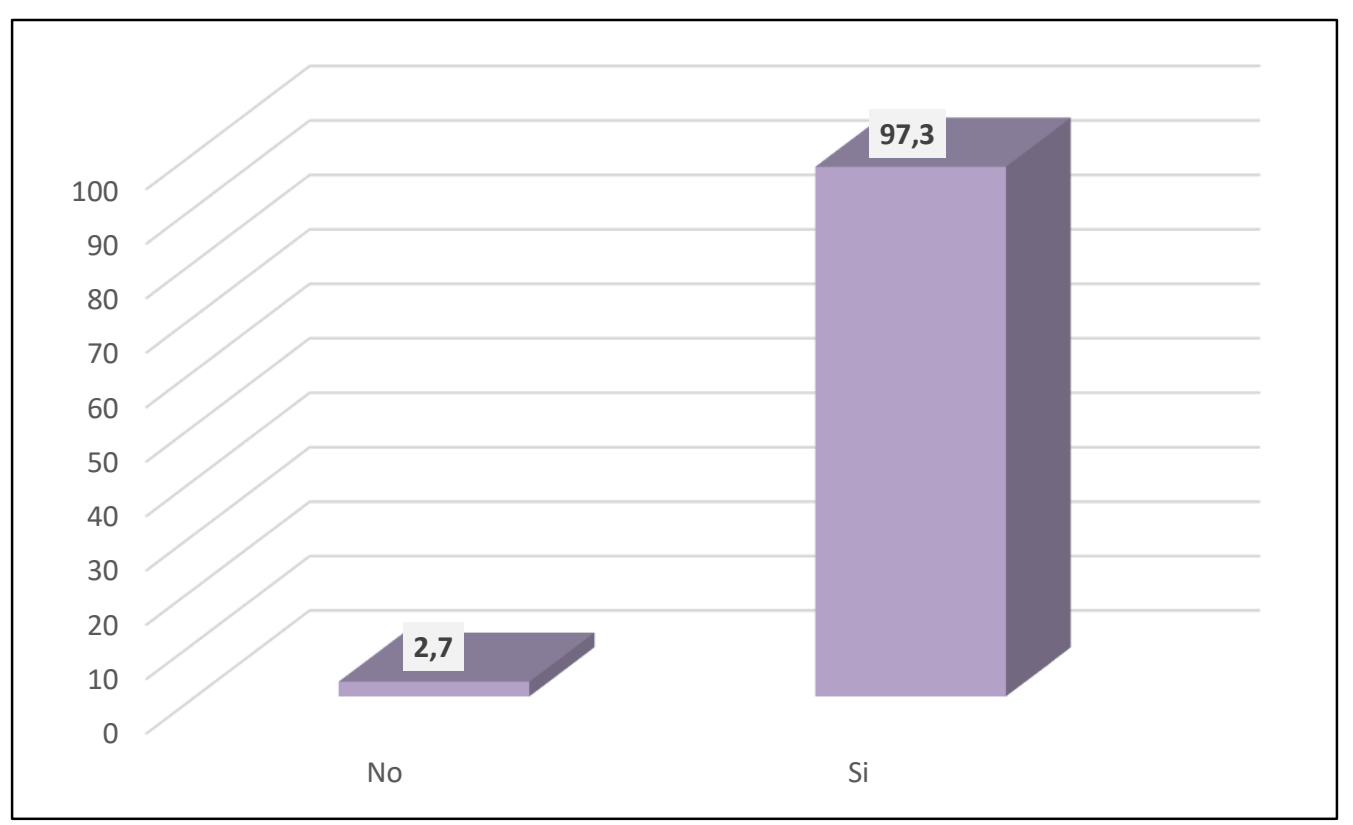

Gráfico 3. Resultados de pruebas realizadas en pacientes para determinar si el software permitió realizar preguntas en el Centro de Salud de Carmen Alto.

Fuente: elaboración propia.

Se observa que en el $97.3 \%$ de los pacientes, el software traductor permitió registrar los síntomas de los pacientes, lo que demuestra ser un buen indicador para afirmar que sí permite registrar los síntomas de los pacientes.

Segundo: resultado del diagnóstico de pacientes de acuerdo al médico quechua hablante y al médico no quechua hablante

Para determinar si el software determinaba efectividad en el diagnóstico de los pacientes, se hizo la prueba en una muestra intencionada de 96 pacientes en el Centro de Salud de Carmen Alto, Ayacucho. 
La prueba consistió en medir el nivel de concordancia entre los diagnósticos de los pacientes elaborados por dos médicos: uno que es quechua hablante (sin software) y otro no quechua hablante (con el software). Para medir dicha concordancia se hizo uso del estadístico de índice de Kappa de Cohen.

Para ello se planteó las siguientes hipótesis estadísticas:

Ho: No existe concordancia entre el diagnóstico del médico quechua hablante y el diagnóstico del médico no quechua hablante.

H1: Existe concordancia entre el diagnóstico del médico quechua hablante y el diagnóstico del médico no quechua hablante.

Tabla 11. Resultado de diagnóstico de pacientes de acuerdo al médico quechua hablante y no quechua hablante.

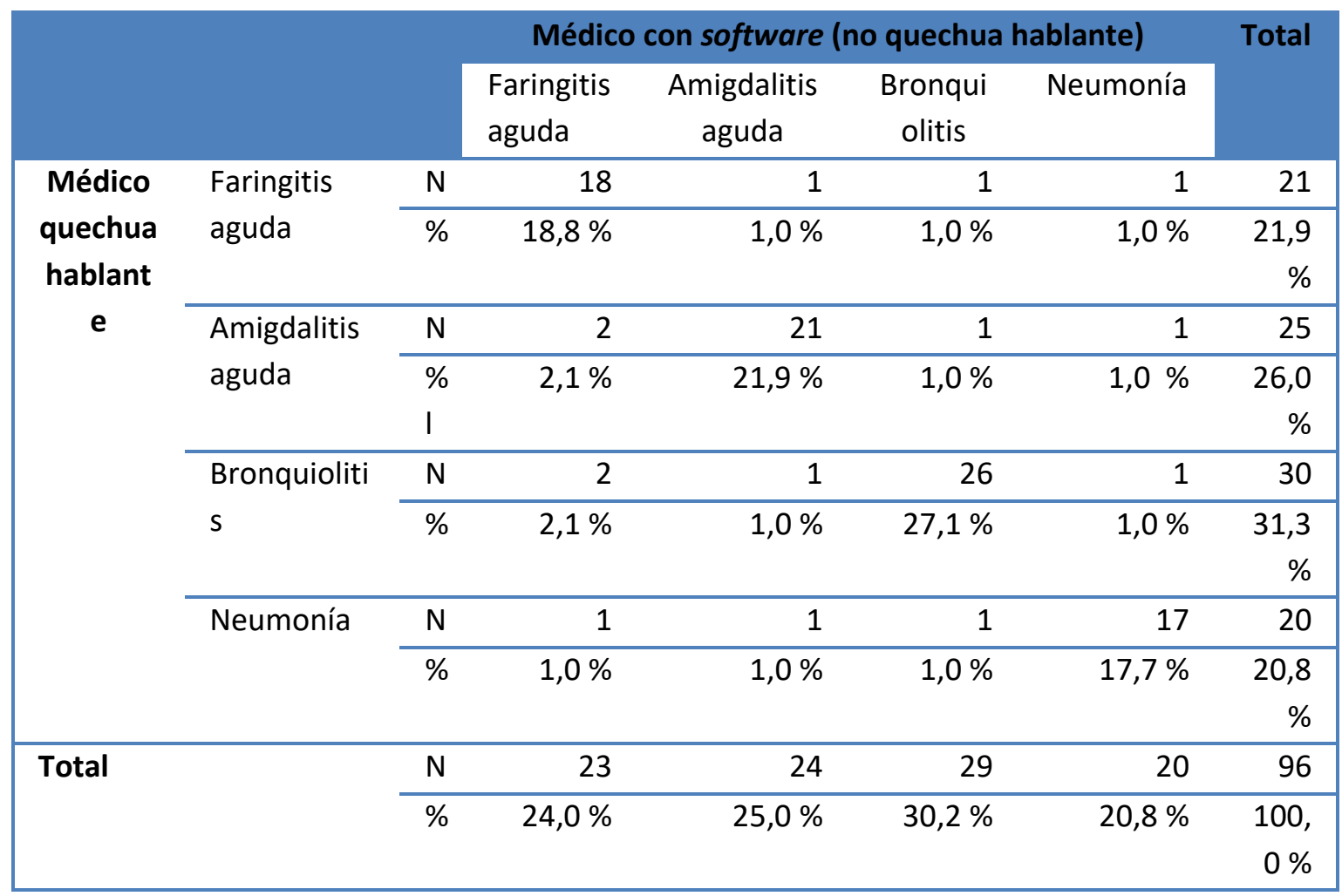

Fuente: elaboración propia. 
Tabla 12. Medidas siméticas.

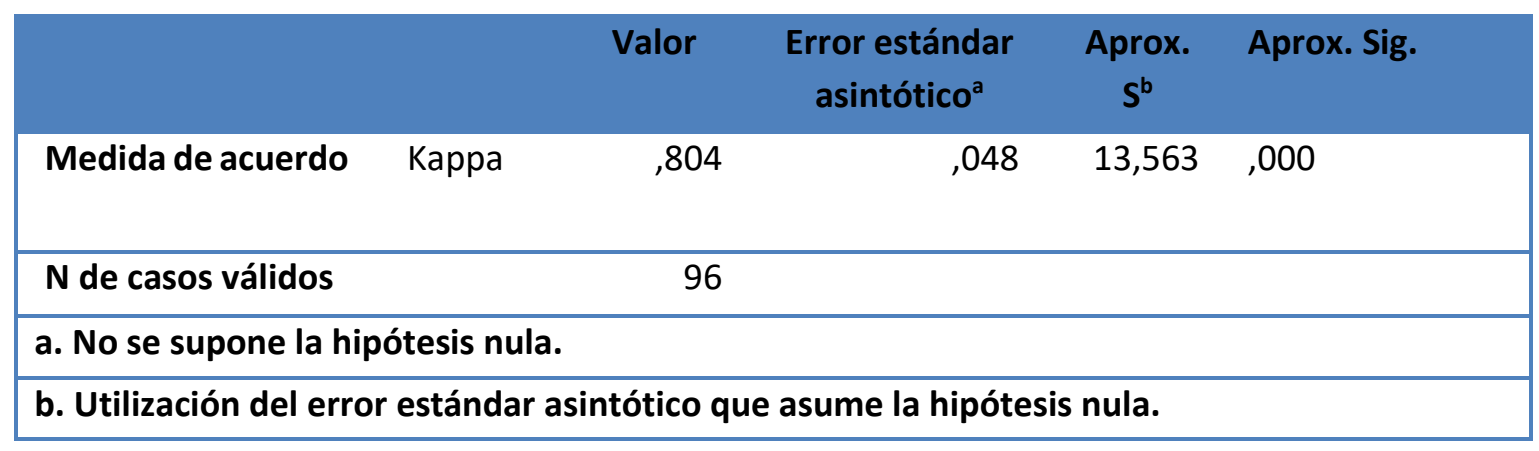

Fuente: elaboración propia.

En la tabla de resultado del diagnóstico de pacientes de acuerdo al médico quechua hablante y no quechua hablante, se puede apreciar que en el 27,1 \% de los diagnósticos los médicos coincidieron que dichos pacientes tienen bronquiolitis. En el $18.8 \%$ de los casos coinciden y diagnostican faringitis aguda, mientras que en 2.1 \% de los casos no coincidieron, ya que el médico quechua hablante diagnosticó amigdalitis aguda y el médico no quechua hablante diagnosticó faringitis aguda.

A continuación, aparecen los resultados propios del análisis de la prueba del índice de Kappa para datos pareados (datos que corresponden al mismo individuo, pero observados por 2 médicos diferentes) en el número de pacientes diagnosticados (96), y finalmente, la probabilidad de error tipo I ( $P$-valor $=0,000)$ asociada al índice de Kappa de Cohen $(0,804)$.

\section{Decisión:}

Como el P-valor es menor de 0,05 entonces se rechaza la Ho, lo cual indica que podemos afirmar a un nivel de confianza al 95\% que existe concordancia casi perfecta (de acuerdo a la valoración del coeficiente de Kappa de acuerdo a Landis y Koch (1997) entre el médico quechua hablante y el médico no quechua hablante.

La prueba de concordancia o armonía entre jueces se encuentra en el protocolo de las características psicométricas de la fiabilidad de un instrumento de medida, y de acuerdo a la existencia de una concordancia casi perfecta entre los médicos, se puede indicar que el software traductor móvil es altamente confiable (buena, de acuerdo a la valoración del índice de Kappa) para el uso en el diagnóstico de los pacientes por parte de los médicos no quechua hablantes. 


\section{CONCLUSIONES}

a) Con un alto nivel de confianza, se precisa que el traductor móvil para asistencia médica a pacientes quechua hablantes permite realizar preguntas satisfactoriamente.

b) Con un alto nivel de confianza, se demuestra que el traductor móvil para asistencia médica a pacientes quechua hablantes permite el registro de síntomas de pacientes satisfactoriamente.

c) Con el índice de Kappa de Cohen de 0,804, se determina que el traductor móvil para asistencia médica es altamente confiable (buena, de acuerdo a la valoración del índice de Kappa) para el diagnóstico de los pacientes por parte de los médicos no quechua hablantes, con un nivel de confianza al $95 \%$.

d) Finalmente, se afirma que es posible desarrollar un software traductor móvil para realizar un diagnóstico adecuado a pacientes quechua hablantes a través de una comunicación fluida.

\section{REFERENCIAS BIBLIOGRÁFICAS}

Baird, S. (2002). Teach Yourself Extreme Programming in 24 Hours. Primera edición. Estados Unidos.

Beck, K. (1999). Extreme programins Explained. Primera Edición. USA. Addison-Wesley urb Co.

Bernal C. (2010). Metodología de la investigación. Tercera Edición. Pearson Educación, Colombia.

Cárdenas A.M, Godoy D.D. (2008). Estrategias de comunicación. Pontificia Universidad Javeriana. Colombia.

Carrasco D. S. (2005). Metodología de la investigación científica. Primera edición. Editorial San Marcos. Lima, Perú.

Computerhope.

(2016).

Recuperado

de:

<http://www.computerhope.com/jargon/t/translator.htm/>.

Date, C.J. (2001). An Introduction to Database Systems. Séptima edición. México. Pearson Educación.

De la Cruz, T. M. (2003). La traducción de textos turísticos: propuesta de clasificación y análisis de muestras.

Recuperado

de:

<http://apps.who.int/iris/bitstream/10665/37500/1/WHO TRS 176 spa.pdf/>.

DSIIC-Universidad Politécnica de Valencia. (2006). Metodologías Agiles para el Desarrollo de Software: Extreme Programming (XP). Valencia, España.

Huayhua, M. (2006). Discriminación y exclusión: políticas públicas de salud y poblaciones indígenas. Disponible: <http://archivo.iep.pe/textos/DDT/DDT147.pdf/>. [05 de marzo del 2016].

Donabedian, A. (1966). Evaluating the quality of medical care. The Milbank Memorial Fund Quarterly, 44 (3), 166-203. Publicado nuevamente en junio 2005, 83 (4), 691-729. 
Donabedian, A. (1984). La Calidad de la atención médica, definición y métodos de evaluación. Editorial La Prensa Médica Mexicana.

Jeffries, R., Anderson, A. y C. Hendricksopn. (2001). Extreme programming Installed. Primera Edición. Usa. Addison-Wesley urb Co.

Joyanes, L. (1996). Programación orientada a objetos. Madrid, España: McGraw - Hill Interamericana de España S.A.U.

Joyanes, L. y Zahonero, I. (2002). Programación en Java 2: Algoritmos, estructuras de datos y Programación orientada a objetos. Madrid, España: McGraw - Hill Interamericana de España S.A.U.

Lopera, Q. J. (2008). Manual de semiología en quechua. Recuperado de: <http://cmp.org.pe/documentos/librosLibres/manualSemiologiaQuechua/>.

Mobile Marketing Asociation. (2011). Libro Blanco de Apps / Guía de apps móviles. Mobile Marketing Asociation.

Organización Mundial de la salud (1959). Segundo informe del comité de Expertos de Organización de la Asistencia Médic. Ginebra.

Pizzolante N. I. (2004). El Poder de la Comunicación Estratégica. Editorial Pontificia Universidad Javeriana. Bogotá.

Pressman, R. (2002). Ingeniería de software: Un enfoque práctico (5므 Ed.). Madrid, España: McGraw Hill Interamericana S.A.

Priolo, S. (2007). Programación extrema.

Sánchez, C.G. (2006), Seminario de Etnolingüística, un estudio de tres lenguas amerindias: Natuatl, quechua y aimara. Informe Final de Seminario de Grado, Universidad de Chile, Chile. Recuprado de: <http://repositorio.uchile.cl/tesis/uchile/2006/gomez m2/sources/gomez m2.pdf/>. [02 de febrero del 2016].

Schmuller, J. (1997). Aprendiendo UML en 24 horas. Naucalpan de Juárez, Mexico: Editorial División Computación.

Servicio Canario de la Salud - España. (2015). Recuperado de: <http://www3.gobiernodecanarias.org/>.

Solis, F.G. (2011). Quechua: una síntesis. Revista cultural electrónica Construyendo nuestra Interculturalidad. Recuperado de: <http://www.interculturalidad.org/numero0607/attachments/article/89/Solis Quechua-Una-sintesis.pdf/>. [20 de enero del 2016].

Tur, J. (1974). Sobre la teoría de la traducción. Centro Virtual Cervantes. Recuperado de: <http://www.colegiotraductores.org.uy/Jaume Tur Sobre teoria de la\%20traduccion.pdf/>. [10 de enero del 2016].

Universal Doctor (2016). Recuperado de: $<\underline{\text { http:} / / w w w . u n i v e r s a l d o c t o r . c o m />. ~}$ 
Valdivia.

(2010).

$<$ http://alertacontraelracismo.pe/wp-

content/uploads/2013/01/Ladiscriminaci\%C3\%B3nenelper\%C3\%BAyelcaso-de-la-salud.pdf/>.

Wasserman, A.I. (2010). Software engineering issues for mobile application development. Proceedings of the FSE/SDP workshop on future of software engineering research, 7-8 de noviembre de 2010 .

Weitzenfeld, A. (2005). Ingeniería de Software Orientado a Objetos con UML, Java e Internet. México, D.F., México: Thomson.

Zurita, B. (1966). Anales de la Facultad de Medicina: Calidad de la Atención de la Salud. Universidad Nacional Mayor de San Marcos. ISSN 1025 - 5583, 57(4). Perú. 199. 